\title{
Nutraceutical approach for the management of cardiovascular risk - a combination containing the probiotic Bifidobacterium longum BB536 and red yeast rice extract: results from a randomized, double-blind, placebo- controlled study
}

Massimiliano Ruscica ${ }^{1}$, Chiara Pavanello ${ }^{1,2}$, Sara Gandini ${ }^{3}$, Chiara Macchi ${ }^{1}$, Margherita Botta ${ }^{1}$, Daria Dall'Orto ${ }^{1}$, Marina Del Puppo ${ }^{4}$, Marco Bertolotti ${ }^{5}$, Raffaella Bosisio ${ }^{6}$, Giuliana Mombelli ${ }^{6}$, Cesare R. Sirtori ${ }^{6}$, Laura Calabresi ${ }^{1,2}$ and Paolo Magni ${ }^{1,7^{*}}$ (D)

\begin{abstract}
Background: Probiotics incorporated into dairy products have been shown to reduce total (TC) and LDL cholesterolemia $(\mathrm{LDL}-\mathrm{C})$ in subjects with moderate hypercholesterolemia. More specifically, probiotics with high biliary salt hydrolase activity, e.g. Bifidobacterium longum BB536, may decrease TC and LDL-C by lowering intestinal cholesterol reabsorption and, combined with other nutraceuticals, may be useful to manage hypercholesterolemia in subjects with low cardiovascular (CV) risk. This study was conducted to evaluate the efficacy and safety of a nutraceutical combination containing Bifidobacterium longum BB536, red yeast rice (RYR) extract (10 mg/day monacolin K), niacin, coenzyme Q10 (Lactoflorene Colesterolo ${ }^{\oplus}$ ). The end-points were changes of lipid CV risk markers (LDL-C, TC, non-HDL-cholesterol (HDL-C), triglycerides (TG), apolipoprotein B (ApoB), HDL-C, apolipoprotein Al (ApoAl), lipoprotein(a) (Lp(a), proprotein convertase subtilisin/kexin type 9 (PCSK9)), and of markers of cholesterol synthesis/absorption.
\end{abstract}

Methods: A 12-week randomized, parallel, double-blind, placebo-controlled study. Thirty-three subjects (18-70 years) in primary CV prevention and low CV risk (SCORE: 0-1\% in 24 and 2-4\% in 9 subjects; LDL-C: 130-200 mg/dL) were randomly allocated to either nutraceutical $(N=16)$ or placebo $(N=17)$.

Results: Twelve-week treatment with the nutraceutical combination, compared to placebo, significantly reduced TC (-16.7\%), LDL-C (- 25.7\%), non-HDL-C (- 24\%) (all $p<0.0001)$, apoB (- 17\%, $p=0.003)$. TG, HDL-C, apoAl, Lp(a), PCSK9 were unchanged. Lathosterol:TC ratio was significantly reduced by the nutraceutical combination, while campesterol:TC ratio and sitosterol:TC ratio did not change, suggesting reduction of synthesis without increased absorption of cholesterol. No adverse effects and a 97\% compliance were observed.

(Continued on next page)

\footnotetext{
* Correspondence: paolo.magni@unimi.it

'Dipartimento di Scienze Farmacologiche e Biomolecolari, Università degli

Studi di Milano, Via Balzaretti 9, 20133 Milan, Italy

${ }^{7}$ IRCCS MultiMedica, Sesto S. Giovanni, Milan, Italy

Full list of author information is available at the end of the article
}

(c) The Author(s). 2019 Open Access This article is distributed under the terms of the Creative Commons Attribution 4.0 International License (http://creativecommons.org/licenses/by/4.0/), which permits unrestricted use, distribution, and reproduction in any medium, provided you give appropriate credit to the original author(s) and the source, provide a link to the Creative Commons license, and indicate if changes were made. The Creative Commons Public Domain Dedication waiver (http://creativecommons.org/publicdomain/zero/1.0/) applies to the data made available in this article, unless otherwise stated. 
(Continued from previous page)

Conclusions: A 12-week treatment with a nutraceutical combination containing the probiotic Bifidobacterium longum BB536 and RYR extract significantly improved the atherogenic lipid profile and was well tolerated by low CV risk subjects.

Trial registration: NCT02689934.

Keywords: Cardiovascular risk, Probiotic, Nutraceutical, Hypercholesterolemia, LDL-cholesterol, Non-HDL cholesterol, Monacolin K

\section{Background}

Atherosclerosis-related cardiovascular (CV) diseases are associated with greater disability, morbidity for concomitant severe conditions and mortality [1]. In addition to some subjects with severe hypercholesterolemia, mostly related to genetic conditions [2], the majority of subjects with low or medium CV risk actually show moderate cholesterol elevation, together with moderate rise of related biomarkers [1]. This CV risk is often underdiagnosed and undertreated, thus representing a significant burden for the individual, especially in combination with unhealthy lifestyle habits [3]. In this field, statins are an established and widely used therapeutic option and their use has led to relevant improvements in the outcome of $\mathrm{CV}$ diseases [4]. However, statins are also well-known to be associated with important side-effects, such as muscle symptoms of different entity [5] and, to a lower extent, de novo diabetes mellitus development [6] indicating the need for additional drug and nutraceutical treatment options.

In all these conditions, nutraceutical approach may be a reasonable option, since in several instances a moderate-intensity (multi)treatment may offer relevant advantages over the no-treatment option or the presence of inadequate adherence to a drug therapy, due for example to adverse effects [7] or even as an add-on to low dose statins in secondary prevention patients intolerant to high dose statin [8]. Several nutraceutical compounds have been evaluated both alone and in combination in the context of moderate dyslipidemia $[9,10]$. Among these, the most widely tested and used are extracts of red yeast rice (RYR), berberine, phytosterols, and stanols [11]. Interestingly, comparative studies between RYR and statins observed a smaller incidence of muscular side effects with the former treatment [12].

Recent evidence indicates that alterations of gut microbiota may be involved in the pathogenesis of systemic diseases related to $\mathrm{CV}$ risk, including hypercholesterolemia [13], suggesting that the use of selected probiotics with specific biological activities may be proposed for these systemic conditions. Indeed, available data suggest that the intake of selected probiotics, incorporated into a food matrix like yogurt or fermented milk, may lead to a significant reduction of total cholesterol (TC) (up to 5.4\%) and low-density lipoprotein-cholesterol (LDL-C) (up to -16\%) [14-16]. More specifically, probiotic strains showing high biliary salt hydrolase (BSH) activity [17, 18], such as Bifidobacterium longum BB536, may contribute to lower circulating TC and LDL-C by reducing intestinal cholesterol reabsorption [19]. As such food matrices may not be very practical for a long-term use, the incorporation of these probiotics into pharmaceutical forms, also in association with other nutraceuticals, may result in better adherence and efficacy for the management of low $\mathrm{CV}$ risk subjects.

The main objective of the present study was the evaluation of the efficacy and safety of a nutraceutical combination containing Bifidobacterium longum BB536, RYR extract, niacin and coenzyme Q10, on the improvement of LDL-C level as the primary end-point, as well as of a set of clinical and experimental markers of $\mathrm{CV}$ risk (secondary end-points).

\section{Methods}

\section{Study design and population}

This was a randomized, double-blind, placebo-controlled, parallel-group trial (RCT) (NCT02689934). It involved 33 subjects in primary $\mathrm{CV}$ prevention, with both low $\mathrm{CV}$ risk and LDL-C in the $130-200 \mathrm{mg} / \mathrm{dL}$ range. The study was performed at the Centro Dislipidemie (ASST Grande Ospedale Metropolitano Niguarda, Milan, Italy) in the period from November 2015 to February 2017, in accordance with the guidelines of the Declaration of Helsinki. The study was approved by the Ethics Committee of ASST Grande Ospedale Metropolitano Niguarda. A written informed consent was obtained from each subject. Sixteen males and 17 females, median aged 57 years $(\mathrm{Q} 1$ $=48$ and $\mathrm{Q} 3=63$ years $)$, with low total CVD risk $(0-1 \%$ in $24 / 33$ subjects $(73 \%)$ and $2-4 \%$ in $9 / 33$ subjects $(27 \%)$ ), as assessed by the SCORE Risk Charts (http://www.heartscore.org/en_GB/) and LDL-C levels of $180(170,196) \mathrm{mg} /$ $\mathrm{dL}$ (median (Q1, Q3)) were recruited for the study (Fig. 1; CONSORT flow diagram). After a run-in period of 4 weeks, patients were randomly assigned to receive, for 12 weeks, either the nutraceutical combination - Lactoflorene Colesterolo ${ }^{\circ}$ (1 sachet/d; granules for oral suspension) containing 1 bn UFC Bifidobacterium longum BB536, RYR extract (10 mg monacolin $\mathrm{K}$ ), $16 \mathrm{mg}$ niacin, $20 \mathrm{mg}$ coenzyme Q10; $n=16$ ) or placebo (1 sachet/d; this latter was identical in taste and appearance to the nutraceutical combination sachet; $n=17$ ) (Fig. 1). Both placebo and 


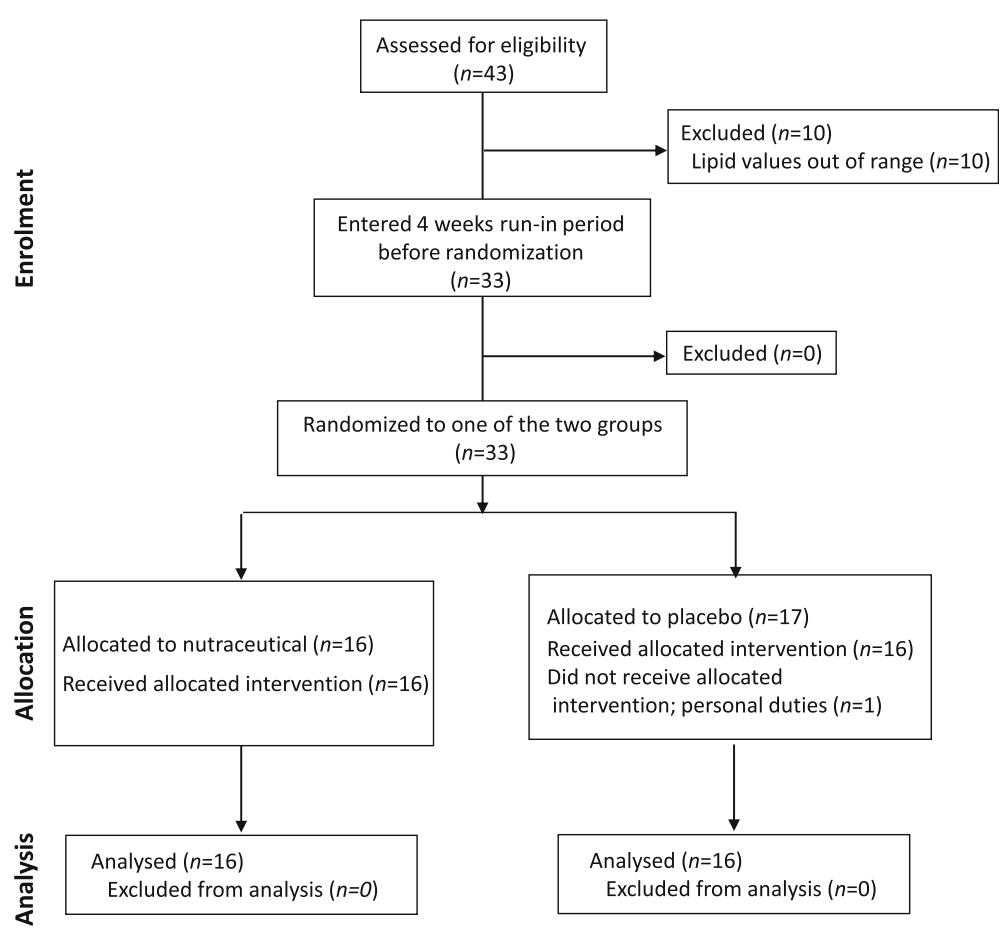

Fig. 1 CONSORT statement flow diagram

active treatment were packaged into a proprietary 2-compartment sachet $\left(\right.$ DUOCAM $\left.^{\circ}\right)$, in order to preserve probiotic integrity. This intervention was followed by a final 4-week follow-up period.

The randomization table was obtained by computergenerated random numbers. Inclusion criteria were: subjects in primary prevention for $\mathrm{CV}$ disease, aged 18-70 years, LDL-C: $130-200 \mathrm{mg} / \mathrm{dL}$, non-smokers. Exclusion criteria were: pregnancy, current or previous smoking, presence of diabetes mellitus, chronic liver disease, renal disease, or severe renal impairment treated with antidiabetic medications or insulin; untreated arterial hypertension; obesity (body mass index - BMI- $\geq 30 \mathrm{~kg} / \mathrm{m}^{2}$; BMI is calculated as weight divided by height squared); any pharmacological treatments known to interfere with the study treatment; and patients enrolled in another research study in the past 90 days. To estimate the compliance, the study subjects were asked to bring back all the sachets left after the first and the second 45-day period.

\section{Clinical procedures}

At study entry, 5 of 30 patients were on standard antihypertensive treatment, maintained for the entire duration of the study (Additional file 1: Table S1). At the screening visit, subjects were instructed to follow a normocaloric/low-saturated fat diet (Additional file 1: Table S2) and the adherence to this dietary scheme was assessed by phone calls every 2 weeks by the dietitian (RB). Clinical and biochemical evaluations were performed at the beginning and at the end of each treatment period. At all visits, patients underwent a fasting venous blood sampling and a full clinical examination, including the determination of height, body weight, waist circumference (by means of a non-stretchable tape at the umbilical level (standing position), heart rate, and arterial blood pressure. Bioelectric impedance analysis (BIA; ViScan device (Tanita Inc., Tokio, Japan)) was used to assess \% abdominal fat mass (BIA (\%)) and \% visceral fat rating (VFR (\%)), according to previously reported procedures (22). All visits were performed by the same investigator (PM), and all ViScan analyses were conducted by the same operator (RB). Plasma samples were immediately separated by centrifugation, and aliquots immediately stored at $-20^{\circ} \mathrm{C}$ for subsequent assays. Primary endpoint was change in LDL-C by study arms. Secondary end points were changes in TC and other $\mathrm{CV}$ biomarkers (non-HDL-C, triglycerides (TG), HDL-C, apolipoprotein (apo)AI, apoB, lipoprotein(a) (Lp(a)), proprotein convertase subtilisin/kexin type 9 (PCSK9)). Data retrieval, analysis, and manuscript preparation were solely the responsibility of the authors.

\section{Biochemical and immunometric assays}

In each blood sample, TC, TG, HDL-C, apoAI, apoB, $\mathrm{Lp}(\mathrm{a})$, fasting plasma glucose (FPG), uric acid, aspartate aminotransferase (AST), alanine aminotransferase (ALT), gamma-glutamyltranspeptidase (GGT), and creatine phosphokinase (CPK) isoenzymes were measured according to 
standard automated clinical procedure. LDL-C was calculated according to the Friedewald formula. Non-HDL-C was calculated as TC minus HDL-C. Commercial enzyme-linked immunosorbent assay (ELISA) kits were used according to manufacturer's specifications and previously published protocols to quantify PCSK9 [20], fibroblast growth factor (FGF) 19 and FGF21, C-reactive protein (all from R\&D System, MN). In addition, oxidized LDL (oxLDL), and insulin were also measured (Mercodia, Sweden). The homeostasis model assessment of insulin resistance (HOMA-IR) index was calculated as follows: HOMA-IR $=[$ fasting glucose $(\mathrm{mg} / \mathrm{dL}) *$ insulin $(\mathrm{mUI} / \mathrm{L}) / 405]$.

\section{Determination of serum levels of lathosterol and plant sterols}

Deuterated lathosterol $(0.5 \mu \mathrm{g})$ and $5 \alpha$-cholestane $(0.5 \mu \mathrm{g})$ were added to $0.1 \mathrm{~mL}$ serum samples as internal standards for the measurement of lathosterol and dietary sterols (campesterol and sitosterol), respectively. After alkaline hydrolysis with $1 \mathrm{~mL} 1 \mathrm{~N} \mathrm{NaOH}$ in $90 \%$ ethanol at $60{ }^{\circ} \mathrm{C}$ for $90 \mathrm{~min}$ under nitrogen, samples were extracted with petroleum ether, transformed into trimethylsilyl (TMS) derivatives and analysed as described previously [21].

\section{Gas chromatography-mass spectrometry analysis}

Analysis of sterols was carried out under previously described conditions (23) monitoring ions at $\mathrm{m} / \mathrm{z} 372$ for detection of cholestane, m/z 255 and 259 for lathosterol and deuterated lathosterol, and m/z 382 and 396 for campesterol and sitosterol, respectively. Lathosterol, campesterol and sitosterol values were normalized by total cholesterol levels. Calibration curves were prepared spiking serum with fixed amounts of each internal standard and increasing amounts of the above-mentioned sterols and were treated and analysed as the samples. Concentrations were calculated on the basis of the slope of the standard curve and on the peak area ratio (sterol/internal standard) found in the sample.

\section{Sample size calculation}

A group sample size of 16 per arm achieves $80 \%$ power to detect a difference of $20 \mathrm{mg} / \mathrm{mL}$ in absolute changes (12 weeks-0 week) in LDL-C levels $(\mathrm{mg} / \mathrm{mL})$, between the null hypothesis that in both arms the means of change in LDL-C are $10 \mathrm{mg} / \mathrm{mL}$ and the alternative hypothesis that the mean of change in LDL-C in the treatment arms is $-10 \mathrm{mg} / \mathrm{mL}$ [22]. The estimated group standard deviations were $25 \mathrm{mg} /$ $\mathrm{mL}$ per arm, with a significance level of $5 \%$ using a two-sided two-sample t-test.

\section{Statistical analysis}

Results are presented as median and interquartile ranges (Q1 and Q3) for all parameters. Differences in median values between treatment arms at baseline were assessed by Wilcoxon-rank sum test. The difference by treatment arms of absolute changes and percentage changes of biomarkers from baseline [12 weeks treatment - baseline (0 week) ] were expressed as median and interquartile ranges. Differences in change by arms were evaluated by ANCOVA models adjusted for baseline values and uncontrolled confounding factors. Percentage changes were assessed considering $20 \%$ as cut-off point of reduction [22]. Chi-square test and multivariate logistic regression models were applied to evaluate the difference between arms in frequencies of subjects with a $20 \%$ reduction. Values of TC, HDL-C, LDL-C and TG were analyzed by repeated-measure ANCOVA models. Mixed effects models are adjusted for baseline value and include as fixed effects: time, treatment arms and age. Residual plots assessed the validity of the assumptions of the models. Least square means are obtained from the full model, adjusted for baseline values, and are presented by time and treatment arms. All tests are 2 -sided, and $P$ values 0.05 are considered statistically significant. Statistical analysis was performed by using the SAS Software version 9.3 (SAS, NC).

\section{Results}

\section{Study population}

All patients were in primary $\mathrm{CV}$ prevention and free from liver/kidney disorders potentially affecting the response to treatment and were not on any drug affecting lipid/lipoproteins or glycaemic profile, including thiazolidinediones or corticosteroids. The baseline clinical and biochemical data indicate that the study subjects showed low CV risk, with $73 \%$ of subjects with a SCORE risk of $0-1 \%$. Median TC was $271(247,288) \mathrm{mg} / \mathrm{dL}$ and LDL-C was $180(170,196) \mathrm{mg} / \mathrm{dL}$ (median (Q1, Q3)) (Table 1). TG, HDL-C, body weight and BMI, waist circumference and blood pressure were within the reference range [23, 24]. Primary and secondary end points and any other clinical parameter at baseline did not differ between the nutraceutical combination group and the placebo group (Table 1).

\section{Effect of nutraceutical treatment on biomarkers of CV risk} After 12 weeks, in the nutraceutical combination group, compared to placebo, we observed significant changes of the main atherogenic lipid parameters. LDL-C was reduced by $45 \mathrm{mg} / \mathrm{dL}(p<0.0001)$, corresponding to a $25.7 \%$ reduction, TC decreased by $45 \mathrm{mg} / \mathrm{dL}(p<0.0001)$, a $-16.7 \%$, apoB by $27 \mathrm{mg} / \mathrm{dL}(p=0.003)$, corresponding to $\mathrm{a}-17 \%$ decrease, and non-HDL-C by $45 \mathrm{mg} / \mathrm{dL}(p<$ 
Table 1 Main baseline clinical and biochemical characteristics of the study population

\begin{tabular}{|c|c|c|}
\hline & Baseline values & $\begin{array}{l}\text { Difference between arms } \\
\text { at baseline ( } P \text {-value) }\end{array}$ \\
\hline Age (years) & $57(48,63)$ & 0.01 \\
\hline Weight (kg) & $65(62,78)$ & 0.43 \\
\hline $\mathrm{BMI}\left(\mathrm{kg} / \mathrm{m}^{2}\right)$ & $24(21,27)$ & 0.89 \\
\hline WC (cm) & $88(84,94)$ & 0.91 \\
\hline BIA (\%) & $31(27,40)$ & 0.99 \\
\hline VFR (\%) & $11(8,12)$ & 0.91 \\
\hline $\mathrm{SBP}(\mathrm{mmHg})$ & $120(120,130)$ & 0.54 \\
\hline $\mathrm{DBP}(\mathrm{mmHg})$ & $80(80,80)$ & 0.88 \\
\hline $\mathrm{HR}(\mathrm{bpm})$ & $64(60,68)$ & 0.24 \\
\hline $\mathrm{TC}(\mathrm{mg} / \mathrm{mL})$ & $271(247,288)$ & 0.51 \\
\hline LDL-C (mg/mL) & $180(170,196)$ & 0.29 \\
\hline $\mathrm{HDL}-\mathrm{C}(\mathrm{mg} / \mathrm{mL})$ & $60(43,77)$ & 0.51 \\
\hline Non-HDL-C (mg/mL) & $210(193,228)$ & 0.24 \\
\hline $\mathrm{TG}(\mathrm{mg} / \mathrm{mL})$ & $115(94,150)$ & 0.79 \\
\hline apoAl (mg/dL) & $118(95,133)$ & 0.28 \\
\hline apoB (mg/dL) & $146(134,155)$ & 0.07 \\
\hline oxLDL (U/L) & $76.6(69.7,86.7)$ & 0.08 \\
\hline $\mathrm{Lp}(\mathrm{a})(\mathrm{mg} / \mathrm{dL})$ & $6(4,12)$ & 0.32 \\
\hline PCSK9 (ng/dL) & $341(285,403)$ & 0.81 \\
\hline $\mathrm{FPG}(\mathrm{mg} / \mathrm{dL})$ & $95(89,98)$ & 0.97 \\
\hline Insulin (mUI/L) & $3.38(2.46,4.92)$ & 0.64 \\
\hline HOMA-IR & $0.75(0.57,1.15)$ & 0.72 \\
\hline FGF19 (pg/mL) & $227(173,337)$ & 0.87 \\
\hline FGF21 (pg/mL) & $179(119,229)$ & 0.37 \\
\hline
\end{tabular}

Statistically significant $\mathrm{P}$-values are indicated in bold

$B M I$ body mass index, WC waist circumference, $B I A$ bioelectrical impedance analysis, VFR visceral fat rating, SBP Systolic blood pressure, $D B P$ diastolic blood pressure, $H R$ heart rate, $T C$ total cholesterol, $L D L-C$ low-density lipoprotein cholesterol, HDL-C high-density lipoprotein cholesterol, TG triglycerides, apoA-I apolipoprotein $\mathrm{A}-\mathrm{I}$, apoB apolipoprotein $\mathrm{B}$, oxLDL oxidize LDL, Lp(a) lipoprotein (a), PCSK9 proprotein convertase subtilisin/kexin type 9, FPG fasting plasma glucose, sICAM-1 soluble intercellular adhesion molecular 1 , HOMA-IR Homeostatic Model Assessment of Insulin Resistance, FGF fibroblast growth factor

0.0001 ), a - 24\% decrease (Table 2). Similar changes were already achieved after 6 weeks of treatment and returned to baseline values after withdrawing the nutraceutical treatment for 4 weeks (data not shown). At the end of the treatment period, circulating oxLDL levels were slightly but significantly reduced by the nutraceutical combination. ApoAI, HDL-C, TG, Lp(a), PCSK9 plasma levels were unchanged in both treatment groups (Table 2). In the placebo group, no significant variations in the circulating biochemical parameters were found. Body weight, BMI, waist circumference, systolic and diastolic blood pressure and heart rate were not affected in any of the two arms (Table 2).

\section{Effects of nutraceutical treatment on cholesterol metabolism}

In order to assess whether the nutraceutical-induced reduction of LDL-C and TC was due to changes in cholesterol synthesis and/or intestinal absorption, the supposed mechanisms of action of RYR extract and Bifidobacterium longum BB536, respectively. The circulating levels of lathosterol, marker of cholesterol synthesis, and of the dietary plant sterols beta-sitosterol and campesterol, markers of intestinal cholesterol absorption, were thus measured. In the nutraceutical combination group, compared to the placebo group, lathosterol:TC was significantly reduced by $-24 \% \quad(p=0.0206)$, whereas campesterol:TC and beta-sitosterol:TC were unchanged (Table 3).

\section{Safety, tolerability and compliance}

Treatment with Lactoflorene Colesterolo ${ }^{\oplus}$ was well tolerated by all participants, who did not report any significant side effects, including gastrointestinal tract or neuromuscular symptoms. No changes in liver and kidney function were observed. Moreover, we observed no changes of FPG, insulin, HOMA-IR, FGF19 and FGF21 in both treatment groups (Table 2). The study subjects showed a high (97\%) compliance to both treatments.

\section{Discussion}

This double-blind RCT conducted in subjects with low $\mathrm{CV}$ risk was aimed at exploring the efficacy and safety of a novel nutraceutical association, combining the Bifidobacterium longum BB536, a probiotic with high BSH activity (18), with a RYR extract, niacin and coenzyme Q10 (Lactoflorene Colesterolo ${ }^{\oplus}$ ). This nutraceutical combination is well accepted, safe and effective in terms of significant improvement of the atherogenic lipid profile. The primary endpoint was met with a $-25.7 \%$ drop in LDL-C; significant reductions were also found for TC (-16.7\%), ApoB (-17\%) and non-HDL-C (-24\%). Since a similar reduction of the lipid atherogenic markers was already achieved after 6 weeks of treatment, this could be a practical timeframe for assessing the individual response in the clinics. The LDL-C goal recommended by the EAS guidelines (LDL-C $<115 \mathrm{mg} / \mathrm{dL}$ ) [25] was reached by $4 / 16$ subjects, whereas in $7 / 16$ participants LDL-C was lower than $130 \mathrm{mg} / \mathrm{dL}$, after nutraceutical treatment, although an expected variability due to individual responsiveness has been observed. The efficacy of this nutraceutical combination in terms of improvement of clinical lipid markers is comparable or even better than that of several other widely used nutraceuticals, evaluated by RCT studies [26-28].

The intake of selected probiotics incorporated into milk or milk derivatives, as mentioned before, has been found to improve the lipid profile of moderately 
Table 2 Summary of primary and secondary end points

\begin{tabular}{|c|c|c|c|c|c|}
\hline & \multicolumn{2}{|l|}{ Placebo } & \multicolumn{2}{|l|}{ Nutraceutical } & \multirow{2}{*}{$\begin{array}{l}\text { Difference of changes } \\
\text { between arms } \\
\text { P-value }\end{array}$} \\
\hline & Baseline & 12 weeks & Baseline & 12 weeks & \\
\hline Weight (kg) & $65(64,79)$ & $66(63,80)$ & $63(59,75)$ & $64(58,74)$ & 0.57 \\
\hline $\mathrm{BMI}\left(\mathrm{kg} / \mathrm{m}^{2}\right)$ & $24(21,27)$ & $24(21,27)$ & $24(22,27)$ & $24(22,27)$ & 0.72 \\
\hline WC $(\mathrm{cm})$ & $88(84,94)$ & $87(82,91)$ & $86(83,94)$ & $91(82,96)$ & 0.74 \\
\hline BIA (\%) & $31(26,41)$ & $31(27,40)$ & $32(27,37)$ & $30(26,38)$ & 0.12 \\
\hline VFR (\%) & $10(7,12)$ & $11(8,13)$ & $11(8,12)$ & $11(8,12)$ & 0.83 \\
\hline $\mathrm{SBP}(\mathrm{mmHg})$ & $125(110,130)$ & $120(110,128)$ & $120(120,130)$ & $120(115,130)$ & 0.95 \\
\hline $\mathrm{DBP}(\mathrm{mmHg})$ & $80(80,80)$ & $80(70,80)$ & $80(80,80)$ & $80(80,83)$ & 0.46 \\
\hline $\mathrm{HR}(\mathrm{bpm})$ & $65(64,68)$ & $64(60,67)$ & $64(60,68)$ & $65(62,71)$ & 0.23 \\
\hline $\mathrm{TC}(\mathrm{mg} / \mathrm{mL})$ & $271(256,289)$ & $267(259,293)$ & $271(239,285)$ & $208(201,263)$ & $<.0001$ \\
\hline $\mathrm{LDL}-\mathrm{C}(\mathrm{mg} / \mathrm{mL})$ & $189(174,198)$ & $183(171,202)$ & $177(167,193)$ & $136.5(118,151.5)$ & $<.0001$ \\
\hline $\mathrm{HDL}-\mathrm{C}(\mathrm{mg} / \mathrm{mL})$ & $56(48,65)$ & $58.5(50,68)$ & $71(43,81)$ & $71(48,88)$ & 0.97 \\
\hline Non-HDL-C (mg/mL) & $215(198,232)$ & $217(199,232)$ & $206(189,214)$ & $198(182,208)$ & $<.0001$ \\
\hline $\mathrm{TG}(\mathrm{mg} / \mathrm{mL})$ & $113(94,127)$ & $112(99,148)$ & $127(93,156)$ & $106(80,124)$ & 0.66 \\
\hline apoAl (mg/dL) & $113.5(97.5,126.5)$ & $110(94.5,120)$ & $128(93,139)$ & $128(100,151)$ & 0.24 \\
\hline apoB (mg/dL) & $143.5(134,148)$ & $135(133,151)$ & $155(137,158)$ & $118(112,131)$ & 0.003 \\
\hline oxLDL (U/L) & $71.2(66.7,84.0)$ & $76.4(71.1,99.5)$ & $78.3(74.6,129.0)$ & $81.0(64.5,114.1)$ & 0.014 \\
\hline Lp(a) (mg/dL) & $4.5(2,15.5)$ & $4(2,17.5)$ & $7(4.5,12)$ & $9(4,14.5)$ & 0.20 \\
\hline PCSK9 (ng/dL) & $341(294,375)$ & $324(259,360)$ & $341(294,375)$ & $346(302,366)$ & 0.38 \\
\hline FPG (mg/dL) & $95(90,97)$ & $93(87,101)$ & $93(89,101)$ & $95(92,103)$ & 0.20 \\
\hline Insulin (mUI/L) & $3.23(2.64,5.24)$ & $3.04(2.31,6.51)$ & $3.38(2.24,4.92)$ & $3.01(2.38,5.12)$ & 0.78 \\
\hline HOMA-IR & $0.73(0.59,1.23)$ & $0.69(0.51,1.64)$ & $0.77(0.52,1.15)$ & $0.77(0.49,1.24)$ & 0.92 \\
\hline FGF19 (pg/mL) & $233(166,337)$ & $261(164,305)$ & $218(177,299)$ & $221(183,374)$ & 0.98 \\
\hline FGF21 (pg/mL) & $185(72,227)$ & $148(77,263)$ & $179(158,364)$ & $161(66,309)$ & 0.11 \\
\hline
\end{tabular}

Data are shown as median (1st quartile, 3rd quartile)

Statistically significant $\mathrm{P}$-values are indicated in bold

$B M I$ body mass index, WC waist circumference, $B I A$ bioelectrical impedance analysis, VFR visceral fat rating, SBP Systolic blood pressure, $D B P$ diastolic blood pressure, $H R$ heart rate, $T C$ total cholesterol, $L D L-C$ low-density lipoprotein cholesterol, $H D L-C$ high-density lipoprotein cholesterol, $T G$ triglycerides, apoA-I apolipoprotein $\mathrm{A}-\mathrm{I}, a p o B$ apolipoprotein $\mathrm{B}$, oxLDL oxidize LDL, $L p(a)$ lipoprotein (a), PCSK9 proprotein convertase subtilisin/kexin type 9, FPG fasting plasma glucose, sICAM-1 soluble intercellular adhesion molecular 1, HOMA-IR Homeostatic Model Assessment of Insulin Resistance, FGF fibroblast growth factor

hypercholesterolemic subjects, with a significant $(-5 /$ $18 \%)$ reduction of $\mathrm{TC}$ and $\mathrm{LDL}-\mathrm{C}[15,19]$, indicating that this approach is relevant and needs to be further pursued. In these studies, however, the amount of food containing the probiotics was quite high (300-350 g or $\mathrm{mL}$ of fermented milk or yogurt per day), making such approach less practical for long-term treatments. On the other hand, we show here that is reasonably simpler, safe and even more effective, for a long-term treatment, to propose a nutraceutical combination containing a probiotic in a specific pharmaceutical form (granules for oral suspension), packaged into a 2-compartment sachet to preserve probiotic integrity. Interestingly, the extent of LDL-C modifications with this nutraceutical combination is similar to the average reduction observed in some of the earlier statin clinical trials, reported in the

Table 3 Determination of serum levels of lathosterol and plant sterols

\begin{tabular}{|c|c|c|c|c|c|}
\hline & \multicolumn{2}{|l|}{ Placebo } & \multicolumn{2}{|l|}{ Nutraceutical } & \multirow{2}{*}{$\begin{array}{l}\text { Difference of changes } \\
\text { between arms } \\
P \text {-value }\end{array}$} \\
\hline & Baseline & 12 weeks & Baseline & 12 weeks & \\
\hline Lathosterol:TC & $0.78(0.62,0.84)$ & $0.72(0.6,0.82)$ & $0.71(0.52,1.01)$ & $0.57(0.41,0.8)$ & 0.0206 \\
\hline Campesterol:TC & $1.45(0.78,1.76)$ & $1.38(0.94,2.12)$ & $1.18(0.84,1.79)$ & $1.41(1.04,1.92)$ & 0.37 \\
\hline Beta-sitosterol:TC & $1.80(1.00,2.56)$ & $1.67(1.28,2.14)$ & $1.62(0.94,2.44)$ & $1.89(1.51,2.54)$ & 0.47 \\
\hline
\end{tabular}

Data are shown as median (1st quartile, 3rd quartile), $P$-values are adjusted for age and baseline values. TC Total Cholesterol Statistically significant $P$-values are indicated in bold 
CTT meta-analysis [29]. Clinical trials with statins and, in general, with lipid-modifying drugs, included very large cohorts and long-term follow-ups and enabled to appreciate the reduction of major adverse $\mathrm{CV}$ events. This important aspect has not yet been addressed by clinical trials with nutraceuticals and remains a relevant challenge for the future. The effects of the combination of another probiotic (L. fermentum ME-3) with RYR and other active components on the lipid profile has also been recently evaluated in an open-label preliminary study, leading to the observation of a significant $-18 \%$ reduction of LDL-C [30]. The use of this nutraceutical combination was associated with a slight reduction of oxLDL levels. Interestingly, the circulating levels of PCSK9, the main regulator of LDL-C, were not affected by this nutraceutical combination, possibly counteracting the known stimulatory effect of RYR alone on this protein [31,32]. Another RCT evaluating a nutraceutical mix including RYR titrated, like in the present study, at $10 \mathrm{mg} /$ day monacolin $\mathrm{K}$, and the antioxidant compound hydroxytyrosol, reported a significant $-20 \%$ reduction of oxLDL [33].

The potential advantage of this nutraceutical combination is that it is supposed to activate two separate mechanisms to promote LDL-C and TC reduction: inhibition of cholesterol synthesis in the liver by RYR extract and reduction of intestinal cholesterol absorption by the Bifidobacterium longum BB536, through its high BSH activity. The analysis of circulating levels of sterols allowed to estimate the relative contribution of the two mechanisms to the observed LDL-C and TC reduction. As expected, a marked lathosterol:TC reduction indicates a strong inhibition of cholesterol synthesis. We found no changes of campesterol:TC and sitosterol:TC upon active treatment. While this may be viewed as a negative finding, it should be noted that treatment with statins, including lovastatin, structurally identical to monacolin $\mathrm{K}$, in addition to reduce liver cholesterol synthesis, with lathosterol:cholesterol reduction, tends to increase intestinal cholesterol absorption, shown by increased campesterol:cholesterol and betasitosterol:cholesterol ratios [26, 34].

Although no data on specific RYR-sterols interactions are available, since monacolin $\mathrm{K}$ is structurally identical to lovastatin, one may predict that RYR extract may behave like a statin concerning the modulation of circulating sterol levels. Our findings therefore through an indirect mechanism suggest that Bifidobacterium longum BB536 may effectively act to minimize a possible monacolin K-driven increase of cholesterol absorption, as suggested by the unchanged campesterol and beta-sitosterol levels. Future studies should assess the potential usefulness of probiotics like Bifidobacterium longum BB536 on top of cholesterol synthesis inhibitors like statins. This nutraceutical combination appears to be safe also in terms of glucose metabolism, since no changes were observed regarding insulin resistance and FGF19 and FGF21 levels [35, 36].

The strengths of this study include some novel aspects in the field of nutraceuticals for $\mathrm{CV}$ risk: i) the inclusion of a probiotic with a specific biological activity (BSH) in a nutraceutical combination, ii) the evaluation of experimental markers of $\mathrm{CV}$ and metabolic risk, in addition to clinical biomarkers, and iii) the assessment, for the first time, of biomarkers of cholesterol synthesis and absorption in a RCT with nutraceuticals. A limitation of the study is that we could not evaluate additional treatment arms with, for example, the RYR extract or the probiotic alone. On the other end, a nutraceutical formulation may be advantageous (or disadvantageous) because of its complexity and studying each single component in a clinical trial may not be very informative.

\section{Conclusions}

A 12-week treatment with a novel nutraceutical combination containing the probiotic Bifidobacterium longum BB536 and RYR extract was well tolerated by subjects with low CV risk and borderline hypercholesterolemia and resulted in a significant improvement of the proatherogenic lipid profile. The use of nutraceuticals in $\mathrm{CV}$ prevention, as well as in other areas related to chronic diseases like oncology, is currently expanding. Future studies may address the feasibility of "multiple probiotic-only" or "probiotic plus prebiotic" approaches to moderate hypercholesterolemia/CV risk.

\section{Additional file}

Additional file 1: Table S1. Concomitant medications. Table S2. Average daily dietary intake. (DOCX 14 kb)

\section{Abbreviations}

apoAl: Apolipoprotein Al; apoB: Apolipoprotein B; BMl: Body mass index; BSH: Biliary salt hydrolase; CV: Cardiovascular; DBP: Diastolic blood pressure; ELISA: Enzyme-linked immunosorbent assay; FGF: Fibroblast growth factor; FPG: Fasting plasma glucose; HDL-C: High-density lipoprotein cholesterol; HMG-CoA: Hydroxymethylglutaryl Coenzyme A; HOMA-IR: Homeostatic model assessment of insulin resistance; LDL-C: Low-density lipoprotein cholesterol; Lp(a): Lipoprotein (a); N: Number; oxLDL: Oxidized low-density lipoprotein; PCSK9: Proprotein convertase subtilisin/kexin 9; RYR: Red yeast rice; SBP: Systolic blood pressure; TC: Total cholesterol; TG: Triglycerides; TMS: Trimethylsilyl; UFC: Unit forming colonies; WC: Waist circumference

\section{Acknowledgements}

The scientific advice of Dr. Ariella Annoni is gratefully acknowledged.

\section{Funding}

This study was supported by unrestricted grants to Centro Dislipidemie (ASST Grande Ospedale Metropolitano Niguarda, Milan, Italy) and to Università degli Studi di Milano (Milan, Italy) from Montefarmaco OTC S.p.A (Bollate, MI, Italy). The founding sponsors had no role in the design of the study, in the collection, analyses, or interpretation of data, in the writing of the manuscript, and in the decision to publish the results. 


\section{Availability of data and materials}

All data are available all the time.

\section{Authors' contributions}

MR wrote the paper and performed ELISA experiments; CP selected the patients and acted as clinical monitor; SG performed all the statistical analysis; CM, MB and DD performed biochemical analyses; DPM performed sterol analyses; MB critically revised the manuscript; RB was the dietician; GM coordinated the clinical work; LC and CRS critically revised the manuscript; PM conceived and coordinated the study, wrote the paper. All authors reviewed the results and approved the final version of the manuscript.

\section{Ethics approval and consent to participate}

The study was approved by the Ethics Committee of ASST Grande Ospedale Metropolitano Niguarda. A written informed consent was obtained from each subject.

\section{Consent for publication}

All the authors have read and approved the manuscript.

\section{Competing interests}

The authors declare that they have no competing interests.

\section{Publisher's Note}

Springer Nature remains neutral with regard to jurisdictional claims in published maps and institutional affiliations.

\section{Author details}

${ }^{1}$ Dipartimento di Scienze Farmacologiche e Biomolecolari, Università degli Studi di Milano, Via Balzaretti 9, 20133 Milan, Italy. ²Dipartimento di Scienze Farmacologiche e Biomolecolari, Centro E. Grossi Paoletti, Università degli Studi di Milano, Milan, Italy. ${ }^{3}$ Division of Epidemiology and Biostatistics, European Institute of Oncology, Milan, Italy. ${ }^{4}$ Dipartimento di Medicina e Chirurgia, Università degli Studi di Milano Bicocca, Monza, Italy. ${ }^{5}$ Dipartimento di Scienze Biomediche, Metaboliche e Neuroscienze, Università degli Studi di Modena e Reggio Emilia, Modena, Italy. ${ }^{6}$ Centro Dislipidemie, ASST Grande Ospedale Metropolitano Niguarda, Milan, Italy. ${ }^{7}$ IRCCS MultiMedica, Sesto S. Giovanni, Milan, Italy.

Received: 11 September 2018 Accepted: 18 February 2019 Published online: 22 February 2019

\section{References}

1. Joseph P, Leong D, McKee M, Anand SS, Schwalm JD, Teo K, Mente A, Yusuf S. Reducing the global burden of cardiovascular disease, part 1: the epidemiology and risk factors. Circ Res. 2017;121:677-94.

2. Sharifi M, Futema M, Nair D, Humphries SE. Genetic architecture of familial Hypercholesterolaemia. Curr Cardiol Rep. 2017;19:44.

3. De Backer GG. Prevention of cardiovascular disease: much more is needed. Eur J Prev Cardiol. 2018;25:1083-6.

4. Cholesterol Treatment Trialists C, Fulcher J, O'Connell R, Voysey M, Emberson J, Blackwell L, Mihaylova B, Simes J, Collins R, Kirby A, et al. Efficacy and safety of LDL-lowering therapy among men and women: metaanalysis of individual data from 174,000 participants in 27 randomised trials. Lancet. 2015;385:1397-405.

5. Magni P, Macchi C, Morlotti B, Sirtori CR, Ruscica M. Risk identification and possible countermeasures for muscle adverse effects during statin therapy. Eur J Intern Med. 2015;26:82-8.

6. Mach F, Ray KK, Wiklund O, Corsini A, Catapano AL, Bruckert E, De Backer G, Hegele RA, Hovingh GK, Jacobson TA, et al. Adverse effects of statin therapy: perception vs. the evidence - focus on glucose homeostasis, cognitive, renal and hepatic function, haemorrhagic stroke and cataract. Eur Heart J. 2018;39:2526-39.

7. Ward NC, Pang J, Ryan JDM, Watts GF. Nutraceuticals in the management of patients with statin-associated muscle symptoms, with a note on realworld experience. Clin Cardiol. 2018;41:159-65.

8. Marazzi G, Campolongo G, Pelliccia F, Quattrino S, Vitale C, Cacciotti L, Massaro R, Volterrani M, Rosano G. Comparison of low-dose statin versus low-dose statin + Armolipid plus in high-intensity statin-intolerant patients with a previous coronary event and percutaneous coronary intervention (ADHERENCE trial). Am J Cardiol. 2017;120:893-7.
9. Cicero AFG, Colletti A, Bajraktari G, Descamps O, Djuric DM, Ezhov M, Fras Z, Katsiki N, Langlois M, Latkovskis $\mathrm{G}$, et al. Lipid lowering nutraceuticals in clinical practice: position paper from an international lipid expert panel. Arch Med Sci. 2017;13:965-1005.

10. Poli A, Barbagallo CM, Cicero AFG, Corsini A, Manzato E, Trimarco B, Bernini F, Visioli F, Bianchi A, Canzone G, et al. Nutraceuticals and functional foods for the control of plasma cholesterol levels. An intersociety position paper. Pharmacol Res. 2018;134:51-60.

11. Pirro M, Vetrani C, Bianchi C, Mannarino MR, Bernini F, Rivellese AA. Joint position statement on "nutraceuticals for the treatment of hypercholesterolemia" of the Italian Society of Diabetology (SID) and of the Italian Society for the Study of arteriosclerosis (SISA). Nutr Metab Cardiovasc Dis. 2017;27:2-17

12. Becker DJ, Gordon RY, Halbert SC, French B, Morris PB, Rader DJ. Red yeast rice for dyslipidemia in statin-intolerant patients: a randomized trial. Ann Intern Med. 2009;150:830-9 W147-839.

13. Weis M. Impact of the gut microbiome in cardiovascular and autoimmune diseases. Clin Sci (Lond). 2018;132:2387-9.

14. Larkin TA, Astheimer LB, Price WE. Dietary combination of soy with a probiotic or prebiotic food significantly reduces total and LDL cholesterol in mildly hypercholesterolaemic subjects. Eur J Clin Nutr. 2009;63:238-45.

15. Shimizu M, Hashiguchi M, Shiga T, Tamura HO, Mochizuki M. Meta-analysis: effects of probiotic supplementation on lipid profiles in Normal to mildly Hypercholesterolemic individuals. PLoS One. 2015;10:e0139795.

16. He J, Zhang F, Han Y. Effect of probiotics on lipid profiles and blood pressure in patients with type 2 diabetes: a meta-analysis of RCTs. Medicine (Baltimore). 2017;96:e9166.

17. Grill J, Schneider F, Crociani J, Ballongue J. Purification and characterization of conjugated bile salt hydrolase from Bifidobacterium longum BB536. Appl Environ Microbiol. 1995:61:2577-82.

18. Grill JP, Manginot-Durr C, Schneider F, Ballongue J. Bifidobacteria and probiotic effects: action of Bifidobacterium species on conjugated bile salts. Curr Microbiol. 1995;31:23-7.

19. Andrade S, Borges N. Effect of fermented milk containing lactobacillus acidophilus and Bifidobacterium longum on plasma lipids of women with normal or moderately elevated cholesterol. J Dairy Res. 2009;76:469-74.

20. Ruscica M, Ferri N, Macchi C, Meroni M, Lanti C, Ricci C, Maggioni M, Fracanzani AL, Badiali S, Fargion S, et al. Liver fat accumulation is associated with circulating PCSK9. Ann Med. 2016;48:384-91.

21. Del Puppo M, Kienle MG, Petroni ML, Crosignani A, Podda M. Serum 27hydroxycholesterol in patients with primary biliary cirrhosis suggests alteration of cholesterol catabolism to bile acids via the acidic pathway. J Lipid Res. 1998;39:2477-82.

22. Ruscica M, Gomaraschi M, Mombelli G, Macchi C, Bosisio R, Pazzucconi F, Pavanello C, Calabresi L, Arnoldi A, Sirtori CR, Magni P. Nutraceutical approach to moderate cardiometabolic risk: results of a randomized, double-blind and crossover study with Armolipid plus. J Clin Lipidol. 2014;8:61-8.

23. Alberti KG, Eckel RH, Grundy SM, Zimmet PZ, Cleeman Jl, Donato KA, Fruchart JC, James WP, Loria CM, Smith SC Jr, et al. Harmonizing the metabolic syndrome: a joint interim statement of the international diabetes federation task Force on epidemiology and prevention; National Heart, Lung, and Blood Institute; American Heart Association; world heart federation; international atherosclerosis society; and International Association for the Study of obesity. Circulation. 2009;120:1640-5.

24. Catapano AL, Graham I, De Backer G, Wiklund O, Chapman MJ, Drexel H, Hoes AW, Jennings CS, Landmesser U, Pedersen TR, et al. 2016 ESC/EAS guidelines for the Management of Dyslipidaemias. Eur Heart J. 2016;37: 2999-3058.

25. Authors/Task Force M, Catapano AL, Graham I, De Backer G, Wiklund O, Chapman MJ, Drexel H, Hoes AW, Jennings CS, Landmesser U, et al. ESC/ EAS guidelines for the Management of Dyslipidaemias: the task Force for the Management of Dyslipidaemias of the European Society of Cardiology (ESC) and European atherosclerosis society (EAS) developed with the special contribution of the European Assocciation for Cardiovascular Prevention \& Rehabilitation (EACPR). Atherosclerosis. 2016;253:281-344.

26. Kasmas SH, Izar MC, Franca CN, Ramos SC, Moreira FT, Helfenstein T, Moreno RA, Borges NC, Figueiredo-Neto AM, Fonseca FA. Differences in synthesis and absorption of cholesterol of two effective lipid-lowering therapies. Braz J Med Biol Res. 2012;45:1095-101.

27. Cicero AFG, Fogacci F, Bove M, Veronesi M, Rizzo M, Giovannini M, Borghi C. Short-term effects of a combined nutraceutical on lipid level, fatty liver 
biomarkers, hemodynamic parameters, and estimated cardiovascular disease risk: a double-blind, placebo-controlled randomized clinical trial. Adv Ther. 2017;34:1966-75.

28. Fogacci F, Grassi D, Rizzo M, Cicero AFG. Metabolic effect of berberinesilymarin association: a meta-analysis of randomized, double-blind, placebocontrolled clinical trials. Phytother Res. 2019. https://doi.org/10.1002/ptr. 6282. [Epub ahead of print]

29. Collins R, Reith C, Emberson J, Armitage J, Baigent C, Blackwell L, Blumenthal R, Danesh J, Smith GD, DeMets D, et al. Interpretation of the evidence for the efficacy and safety of statin therapy. Lancet. 2016;388: 2532-61.

30. Kullisaar T, Zilmer K, Salum T, Rehema A, Zilmer M. The use of probiotic L. fermentum ME-3 containing Reg'Activ Cholesterol supplement for 4 weeks has a positive influence on blood lipoprotein profiles and inflammatory cytokines: an open-label preliminary study. Nutr J. 2016;15:93.

31. Macchi C, Banach M, Corsini A, Sirtori CR, Ferri N, Ruscica M. Changes in circulating pro-protein convertase subtilisin/kexin type 9 levels experimental and clinical approaches with lipid-lowering agents. Eur J Prev Cardiol. 2019 https://doi.org/10.1177/2047487319831500. [Epub ahead of print].

32. Momtazi AA, Banach M, Pirro M, Katsiki N, Sahebkar A. Regulation of PCSK9 by nutraceuticals. Pharmacol Res. 2017;120:157-169.

33. Verhoeven V, Van der Auwera A, Van Gaal L, Remmen R, Apers S, Stalpaert M, Wens J, Hermans N. Can red yeast rice and olive extract improve lipid profile and cardiovascular risk in metabolic syndrome?: a double blind, placebo controlled randomized trial. BMC Complement Altern Med. 2015;15:52.

34. Uusitupa MI, Miettinen TA, Happonen P, Ebeling T, Turtola H, Voutilainen E, Pyorala K. Lathosterol and other noncholesterol sterols during treatment of hypercholesterolemia with lovastatin alone and with cholestyramine or guar gum. Arterioscler Thromb. 1992;12:807-13.

35. Gasser E, Moutos CP, Downes M, Evans RM. FGF1 - a new weapon to control type 2 diabetes mellitus. Nat Rev Endocrinol. 2017;13:599-609.

36. Lan T, Morgan DA, Rahmouni K, Sonoda J, Fu X, Burgess SC, Holland WL, Kliewer SA, Mangelsdorf DJ. FGF19, FGF21, and an FGFR1/beta-klothoactivating antibody act on the nervous system to regulate body weight and Glycemia. Cell Metab. 2017;26:709-18 e703.

Ready to submit your research? Choose BMC and benefit from:

- fast, convenient online submission

- thorough peer review by experienced researchers in your field

- rapid publication on acceptance

- support for research data, including large and complex data types

- gold Open Access which fosters wider collaboration and increased citations

- maximum visibility for your research: over $100 \mathrm{M}$ website views per year

At BMC, research is always in progress.

Learn more biomedcentral.com/submissions 